\title{
A Stability Result for the Modified Craig-Sneyd Scheme Applied to 2D and 3D Pure Diffusion Equations
}

\author{
Karel in 't Hout and Chittaranjan Mishra \\ Department of Mathematics and Computer Science, University of Antwerp, \\ Middelheimlaan 1, 2020 Antwerp, Belgium
}

\begin{abstract}
The stability of ADI type schemes is considered when applied to two- and three-dimensional pure diffusion equations with mixed derivative terms. We present a first stability result that takes the actual size of the mixed derivative coefficients into account.
\end{abstract}

Keywords: initial-boundary value problems, diffusion equations, finite difference methods, ADI splitting schemes, von Neumann stability PACS: $02.60 . \mathrm{Lj}, 02.70 . \mathrm{Bf}$

\section{INTRODUCTION}

This paper deals with the stability of Alternating Direction Implicit (ADI) schemes when adapted to (semi-discretized) multi-dimensional partial differential equations (PDEs) possessing mixed spatial derivative terms. PDEs of this kind are pervasive, notably, in the field of financial mathematics, see e.g. [4, 5]. ADI schemes form a popular class of numerical solution methods. However, ADI schemes were not originally developed for PDEs with mixed derivative terms, and it is thus important to gain insight in their stability in these applications. Recently, In 't Hout \& Welfert [3] proved positive stability results in the von Neumann sense for a variety of ADI schemes when applied to multidimensional pure diffusion equations with mixed derivative terms. These results are valid without any assumption on the size of the coefficients of the mixed derivatives, except that the diffusion equation is parabolic. In the present paper we introduce a useful refinement of the analysis from [3] that takes the actual size of the mixed derivative coefficients into account. We derive for a specific ADI scheme a first stability result in this new setting.

Consider for $k=2$ or $k=3$ the $k$-dimensional pure diffusion equation with mixed derivative terms

$$
\frac{\partial u}{\partial t}=\sum_{i \neq j} d_{i j} u_{x_{i} x_{j}}+d_{11} u_{x_{1} x_{1}}+d_{22} u_{x_{2} x_{2}}+\cdots+d_{k k} u_{x_{k} x_{k}}
$$

on the spatial domain $[0,1]^{k}$ with given initial condition and periodic boundary condition. Here $D=\left[d_{i j}\right]_{1 \leq i, j \leq k}$ is any given constant real matrix that is always assumed to be symmetric and positive semi-definite, in particular

$$
d_{j j} \geq 0 \quad \text { and } \quad\left|d_{i j}\right| \leq \sqrt{d_{i i} d_{j j}} \text { for } 1 \leq i, j \leq k .
$$

Finite difference discretization on a Cartesian grid of the spatial derivatives in equation (1) leads to initial value problems for large systems of stiff ordinary differential equations (ODEs),

$$
U^{\prime}(t)=A U(t) \quad(t \geq 0), \quad U(0)=U_{0}
$$

with given real matrix $A$ and real vector $U_{0}$. For the efficient numerical solution of semi-discrete systems (3), splitting schemes of the ADI type form an attractive class of methods. The adaptation of ADI schemes in the presence of mixed derivative terms starts with a splitting of the matrix $A$ into

$$
A=A_{0}+A_{1}+\cdots+A_{k},
$$

where $A_{0}$ represents all mixed derivatives and $A_{j}$ (for $1 \leq j \leq k$ ) represents the derivative in the $j$-th spatial direction. We consider in this paper a specific method of the ADI type, called the Modified Craig-Sneyd (MCS) scheme. For any given time step $\Delta t>0$, the MCS scheme defines approximations $U_{n}$ to $U(n \cdot \Delta t)$ successively for $n=1,2,3, \ldots$ by 


$$
\left\{\begin{array}{l}
Y_{0}=U_{n-1}+\Delta t A U_{n-1}, \\
Y_{j}=Y_{j-1}+\theta \Delta t A_{j}\left(Y_{j}-U_{n-1}\right) \quad(j=1,2, \ldots, k), \\
\widehat{Y}_{0}=Y_{0}+\theta \Delta t A_{0}\left(Y_{k}-U_{n-1}\right) \\
\widetilde{Y}_{0}=\widehat{Y}_{0}+\left(\frac{1}{2}-\theta\right) \Delta t A\left(Y_{k}-U_{n-1}\right), \\
\widetilde{Y}_{j}=\widetilde{Y}_{j-1}+\theta \Delta t A_{j}\left(\widetilde{Y}_{j}-U_{n-1}\right) \quad(j=1,2, \ldots, k), \\
U_{n}=\widetilde{Y}_{k}
\end{array}\right.
$$

Here $\theta>0$ denotes any given real parameter. The MCS scheme was introduced and studied in [3] and subsequently examined in $[1,2]$. Taylor expansion yields that the scheme has classical order of consistency equal to two for any $\theta$. An inspection of (5) shows that the $A_{0}$ part is always treated in an explicit way, whereas the $A_{j}$ parts for $1 \leq j \leq k$ are successively treated in an implicit way. The scheme (5) retains the key advantage of traditional ADI schemes: the linear systems are efficiently solved by $L U$ factorization, as the pertinent matrices all possess a small bandwidth.

This paper deals with a von Neumann stability analysis of the MCS scheme. In line with the well-known von Neumann analysis we already assumed a periodic boundary condition and constant coefficients $d_{i j}$ and we next assume also constant mesh widths in the different spatial directions. From this it follows that the $A_{j}(0 \leq j \leq k)$ are Kronecker products of circulant (hence normal) matrices that commute with each other, and are thus simultaneously diagonalizable by a unitary matrix. This implies that stability can be rigorously analyzed by considering the scalar test equation

$$
U^{\prime}(t)=\left(\lambda_{0}+\lambda_{1}+\cdots+\lambda_{k}\right) U(t) \quad(t \geq 0)
$$

with $\lambda_{j}$ corresponding eigenvalues of $A_{j}(0 \leq j \leq k)$. In the case of (6), the MCS scheme becomes the scalar iteration

$$
U_{n}=S_{\theta}\left(z_{0}, z_{1}, \ldots, z_{k}\right) U_{n-1},
$$

where $z_{j}=\Delta t \cdot \lambda_{j}$ and

$$
S_{\theta}\left(z_{0}, z_{1}, \ldots, z_{k}\right)=1+\frac{z_{0}+z}{p}+\theta \frac{z_{0}\left(z_{0}+z\right)}{p^{2}}+\left(\frac{1}{2}-\theta\right) \frac{\left(z_{0}+z\right)^{2}}{p^{2}}
$$

with the notation

$$
z=z_{1}+z_{2}+\cdots+z_{k} \quad \text { and } \quad p=\left(1-\theta z_{1}\right)\left(1-\theta z_{2}\right) \cdots\left(1-\theta z_{k}\right) .
$$

The iteration (7) is stable if

$$
\left|S_{\theta}\left(z_{0}, z_{1}, \ldots, z_{k}\right)\right| \leq 1 \text {. }
$$

In [3] it was shown that for the standard central second-order FD discretization the scaled eigenvalues satisfy

$$
\begin{aligned}
& \text { all } z_{j} \text { are real, } \\
& z_{j} \leq 0 \text { for } 1 \leq j \leq k, \\
& z+z_{0} \leq 0, \\
& \left|z_{0}\right| \leq \sum_{i \neq j} \sqrt{z_{i} z_{j}},
\end{aligned}
$$

and the positive result was proved that if $\theta \geq \frac{1}{3}$ (for $k=2$ ) or $\theta \geq \frac{6}{13}$ (for $k=3$ ) then the stability requirement (9) is fulfilled, and hence, the MCS scheme is unconditionally stable in the von Neumann sense. In the following we derive a useful generalization of this result, which takes the size of the mixed derivative coefficients $d_{i j}(i \neq j)$ into account. 


\section{FORMULATION OF THE MAIN RESULT}

Consider for $\gamma \in[0,1]$ the condition

$$
d_{j j} \geq 0 \quad \text { and } \quad\left|d_{i j}\right| \leq \gamma \sqrt{d_{i i} d_{j j}} \text { for } 1 \leq i, j \leq k .
$$

Clearly the quantity $\gamma$ is a measure for the relative size of the mixed derivative coefficients in the diffusion equation (1). If $\gamma=1$, then the condition (2) is obtained, which admits the largest possible mixed derivative coefficients. On the other hand, if $\gamma=0$, then all mixed derivative coefficients vanish. In financial applications one usually has $0<\gamma<1$. We remark that, by a similar derivation as in [3], if the condition (11) holds then the scaled eigenvalues satisfy

$$
\left|z_{0}\right| \leq \gamma \sum_{i \neq j} \sqrt{z_{i} z_{j}}
$$

The property (12) is obviously stronger than (10d) whenever $\gamma<1$. The interesting question arises, to any given $\gamma$, for which range of parameter values $\theta$ is the MCS scheme unconditionally stable. We have the following neat result.

Theorem 1. Consider equation (1) for $k=2$ or $k=3$ with symmetric positive semi-definite matrix $D$ and periodic boundary condition. Assume (3), (4) is obtained by central second-order FD discretization and splitting as described in Section. Let $\gamma \in[0,1]$ be such that (11) holds. Then the MCS scheme is unconditionally stable when applied to (3), (4) whenever $\theta \geq \max \left\{\frac{1}{4}, \frac{1}{6}(\gamma+1)\right\}$ (for $\left.k=2\right)$ or $\theta \geq \max \left\{\frac{1}{4}, \frac{2}{13}(2 \gamma+1)\right\}($ for $k=3)$.

The above result can be viewed as a generalization to Theorem 2.5 in [3]. The latter theorem is recovered by setting $\gamma$ equal to 1 .

Proof. The proof follows along the lines of [3] and uses the properties (10a)-(10c) and (12) of the scaled eigenvalues. For the sake of brevity, we consider here only the case $k=2$ and shall incorporate the proof for $k=3$ in a future paper.

The stability requirement (9) is equivalent to (13), where

$$
\begin{aligned}
& 2 p^{2}+p z+\left(\frac{1}{2}-\theta\right) z^{2}+[p+(1-\theta) z] z_{0}+\frac{1}{2} z_{0}^{2} \geq 0, \\
& p-\theta z+\frac{1}{2}\left(z_{0}+z\right) \geq 0 .
\end{aligned}
$$

The condition (13a) is fulfilled if the discriminant

$$
\begin{aligned}
\Delta & =[p+(1-\theta) z]^{2}-2\left[2 p^{2}+p z+\left(\frac{1}{2}-\theta\right) z^{2}\right] \\
& =-3 p^{2}-2 \theta p z+\theta^{2} z^{2} \\
& =-(p+\theta z)(3 p-\theta z)
\end{aligned}
$$

is non-positive, and this holds (note that $p \geq 1-\theta z \geq 1$ ). Next consider the condition (13b). Write $y_{j}=\sqrt{-\theta z_{j}}$. Then

$$
p=\left(1+y_{1}^{2}\right)\left(1+y_{2}^{2}\right) \quad \text { and } \quad z=-\frac{1}{\theta}\left(y_{1}^{2}+y_{2}^{2}\right)
$$

and

$$
z+z_{0} \geq z-\left|z_{0}\right| \geq z_{1}+z_{2}-2 \gamma \sqrt{z_{1} z_{2}}=-\frac{1}{\theta}\left(y_{1}^{2}+y_{2}^{2}+2 \gamma y_{1} y_{2}\right)
$$

Using this one readily obtains that if

$$
\left(1+y_{1}^{2}\right)\left(1+y_{2}^{2}\right)+y_{1}^{2}+y_{2}^{2}-\kappa\left(y_{1}^{2}+y_{2}^{2}+2 \gamma y_{1} y_{2}\right) \geq 0
$$

for some $\kappa>0$, then (13b) is fulfilled whenever $\theta \geq 1 /(2 \kappa)$. The inequality (14) can be rewritten as

$$
(2-\kappa)\left(y_{1}-y_{2}\right)^{2}+\left(y_{1} y_{2}+2-\kappa-\kappa \gamma\right)^{2}+1-(2-\kappa-\kappa \gamma)^{2} \geq 0,
$$

which is clearly satisfied when

$$
\kappa \leq 2 \text { and }|2-\kappa-\kappa \gamma| \leq 1 .
$$

The latter condition is equivalent to

$$
\frac{1}{\gamma+1} \leq \kappa \leq \min \left\{2, \frac{3}{\gamma+1}\right\}
$$

Selecting for $\kappa$ the rightmost value of this interval, it follows that (13b) holds whenever $\theta \geq \max \left\{\frac{1}{4}, \frac{1}{6}(\gamma+1)\right\}$. 


\section{REFERENCES}

1. K. J. in 't Hout \& S. Foulon, ADI finite difference schemes for option pricing in the Heston model with correlation, Int. J. Numer. Anal. Mod. 7 (2010) 303-320.

2. K. J. in 't Hout \& C. Mishra, Stability of the Modified Craig-Sneyd scheme for two-dimensional convection-diffusion equations with mixed derivative term, submitted for publication (2010).

3. K. J. in 't Hout \& B. D. Welfert, Unconditional stability of second-order ADI schemes applied to multi-dimensional diffusion equations with mixed derivative terms, Appl. Numer. Math. 59 (2009) 677-692.

4. S. E. Shreve, Stochastic Calculus for Finance II, Springer, New York, 2004.

5. D. Tavella \& C. Randall, Pricing Financial Instruments, Wiley, New York, 2000. 